\title{
Periodontal disease affecting tooth furcations. A review of the treatments available
}

\author{
Arturo Sánchez-Pérez ${ }^{1}, M^{a}{ }^{\text {José Moya-Villaescusa }}{ }^{2}$ \\ ${ }^{1}$ Head of the Peridontics Unit. University of Murcia. Murcia, Spain \\ ${ }^{2}$ Assistant Profesor, Periodontics Unit. University of Murcia. Murcia, Spain
}

Correspondence:

Unidad de Periodoncia. Universidad de Murcia

Clínica Odontológica Universitaria, $2^{a}$ Planta

C/Marqués de los Vélez s/n

30008 - Murcia. Spain

arturosa@um.es

\author{
Sánchez-Pérez A, Moya-Villaescusa $\mathrm{M}^{\mathrm{a} J}$. Periodontal disease affecting \\ tooth furcations. A review of the treatments available. Med Oral Patol \\ Oral Cir Bucal. 2009 Oct 1;14 (10):e554-7. \\ http://www.medicinaoral.com/medoralfree01/v14i10/medoralv14i10p554.pdf

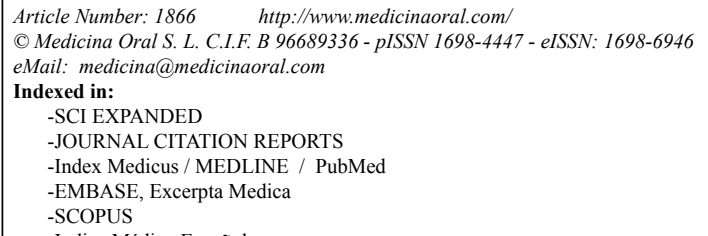

Received: 05/09/2008

Accepted: 31/07/2009

\begin{abstract}
The molars are the teeth that suffer the greatest periodontal destruction in untreated patients. When periodontal disease affects the furcation of a tooth, the chance that it will be lost increases considerably. An increase in the exposed root surface, anatomical peculiarities and irregularities of the furcation surface all favor the growth of bacteria. These problems make it harder for the patient to maintain hygiene, and impede adequate treatment. The treatment of furcations affected by periodontal disease is one of the most difficult problems for the general dentist and periodontist. The motivation of both the attending professional and of the patient are therefore of great importance. No ideal procedure for treating such lesions exists. The present paper reviews those options that are currently available. Long-term research will be needed, along with the development of new techniques, to solve the problem of furcations affected by periodontal disease, possibly including substitution of the affected tooth by an implant.
\end{abstract}

Key words: Furcation defects/therapy, furcation defects/classification, furcation defects/surgery, furcation defects/ diagnosis, prognosis, tooth root/anatomy \& histology, tooth root/pathology.

\section{Introduction}

Periodontal disease is of bacterial origin and is associated with an inflammatory response. The condition takes a chronic course in which both local and systemic factors are of great importance (1). In the posterior dentition, many factors influence the onset and progress of periodontal disease (2), and the loss of insertion of the roots is one of the most important sequelae. Access to periodontal lesions in the furcations is difficult for both patients and dental professionals, and their treatment constitutes an enormous challenge. The loss of affected molars is common. The aim of the present work is to review the epidemiology, diagnosis and treatment of furcations affected by periodontal disease.

\section{Prevalence of furcation lesions}

Periodontal lesions affecting furcations are usually seen in the first upper and lower molars (3), these being the teeth which, over a patient's lifetime, have suffered the longest exposure to the action of plaque. This association means that furcation problems increase with age. When a furcation becomes visible, the risk of losing the affected tooth increases (4).

In the lower molars, the vestibular furcation is the most 
commonly affected furcation, while in the upper molars the vestibular furcation is likewise usually the most commonly affected furcation, followed by the mesiopalatine and distopalatine furcations. One furcation among the first and second upper molars is affected by periodontal disease in $50 \%$ of all patients over 30 years of age (5). The upper first molars are more often affected than the lower first molars (3).

\section{Diagnosis}

The precise diagnosis of periodontal disease affecting a furcation requires evaluation of the patient's clinical history, consultation with other specialists when indicated, a periodontal and radiographic examination, and, if necessary, laboratory tests. The condition of a furcation must be known before a prognosis can be established. The extent of furcation disease can be determined by evaluating the following parameters:

1. Vertical bone loss

2. Horizontal bone loss

3. Both the above

Horizontal bone loss is the most commonly used parameter, and in Europe the classification proposed by Hamp (6) is usually employed (Fig. 1):

- Grade 0: No involvement of the furcation.

- Grade I: The furcation is detectable with a probe, but no more than $1 / 3$ is exposed.

- Grade II: The probe can penetrate more than $1 / 3$ of the furcation, but not pass right through the tooth.

- Grade III: The probe passes from one side of the furcation to the other.
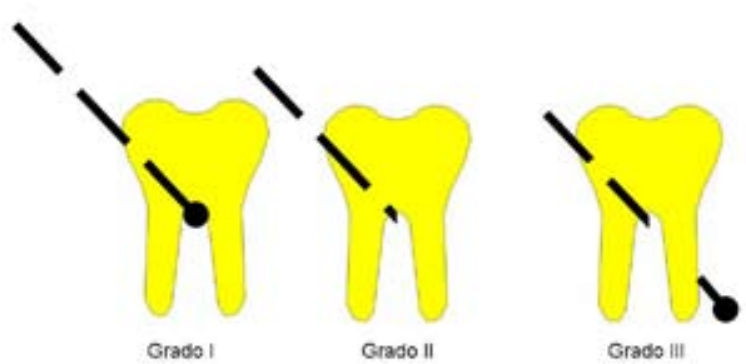

Fig. 1. Classification of different furcations.

A diagnosis can be made using a probe, a conventional periodontal probe, or a specific periodontal instrument such as a Nabers probe. The latter is designed to determine the degree of penetration that can be achieved. Lower vestibular furcations are the easiest type to clinically explore - the hardest type being upper distopalatine furcations.

Knowing the radiotransparency of a furcation is of great help when trying to establish a diagnosis. However, consideration is required of the 'delay' which X-ray images suffer with respect to reality (generally several months), the minimum size necessary to detect a lesion $(0.5 \mathrm{~mm})$, the technical problems of correctly positioning the X-ray plate, and the superimposition of anatomical structures. X-rays also provide a means of monitoring the success of treatment. A long root trunk, a wide furcation and a crown fornix at the cement-enamel junctions are described by some authors as signs of a poor prognosis (7).

Teeth affected with furcation periodontal disease pose a serious problem that compromises and modifies both the treatment plan and the prognosis.

Teeth with Grade III disease are commonly extracted, but even teeth with Grade I and II disease are candidates for extraction when the patient has sufficient bone and the treatment required is long or complicated, and the prognosis uncertain. However, an uncertain outcome does not imply that all teeth with periodontal disease affecting the furcation should be extracted.

\section{Factors associated with furcation periodontal disease}

In order to correctly characterize a furcation, the following factors (many of which are anatomical and biomechanical in nature) should be taken into account (2):

- The size of the furcation

- The length of the root trunk

- Root divergence

- Root fusion

- Root concavity

- Separation of the roots

- The remaining bone (crown/root ratio)

- Mobility

- Occlusion (prematurity and interferences)

- Adherence of the gum

- The ease with which hygiene of the affected furcation can be maintained

- The capacity of the patient to maintain optimum hygiene

In addition, the following should be taken into account: - Age

- Sex

- Degree of hygiene actually maintained

- Smoking

- Parafunctional habits

\section{Differential diagnosis}

Furcation lesions are usually caused by plaque, a consequence of periodontal disease, but also by other factors:

- Occlusal trauma

- Periodontal pulp disease (accessory pulp canal)

- Root fracture

- Invagination of the enamel 
- Enamel pearls

- Developmental grooves

A differential diagnosis is therefore essential.

\section{Treatment of furcation lesions}

Treatment will depend on the extent of the disease, the strategic importance of the affected tooth, and the degree of patient cooperation. All therapy must first begin with periodontal treatment (systemic and basic phases, followed by re-evaluation) and adequate hygiene on the part of the patient. Undoubtedly, the bifurcations constitute one of the zones in the oral cavity where plaque is most difficult to remove. Knowledge of the root morphology and of the topography of the pocket is essential if access to difficult areas is to be gained. It is usually much easier to work with an ultrasound probe or drill than with manual devices.

- Treatment of Grade I diseased furcations

Monitoring of hygiene. Monitoring should ensure that through adequate hygiene the patient is able to prevent a lesion from progressing. Follow-up should be strict, and re-evaluation frequent. If there are no inconveniences, a more interventional strategy can be adopted should the lesion progress.

Debriding with or without a flap but with no modification of the furcation. Both techniques reduce the probing depth in the deepest areas of the lesion. The gain in clinical insertion is reported to improve slightly - but not significantly - when this treatment is performed without a flap (2).

Odontoplasty. The aim of this treatment is to eliminate the opening of the furcation through debridement of the enamel and/or cementation of the furcation. It may be accompanied by osteoplasty. This therapy seeks to improve accessibility and facilitate hygiene, and to remove the bacterial reservoir in the grooves of the furcation pillars. Its drawbacks include the possibility of generating sensitivity, the appearance of secondary caries, progression may not be avoided, and the patient must be highly motivated. It is mainly indicated for use with incipient lesions with very well defined cement-enamel junctions.

Covering of the furcation. This therapy is based on mucogingival root coverage surgery, and involves connective tissue grafts and coronally positioned flaps. In this case, however, it is performed for functional rather than aesthetic reasons. Bone infilling of $18 \%-70 \%$ of the initial volume of the defect has been recorded. Some authors include root detoxification in such therapy, but this does not seem to improve the results obtained. Over the short term, coronally positioned flaps with or without root conditioning, whether accompanied or not by substitutions with freeze-dried, decalcified human bone, may provide good results, but improvements are lost within 4-5 years. The main drawbacks are the risk of lesion progression in the furcation hidden by the covering, and therapeutic failure, which is accompanied by disease progression (8).

- Treatment of Grade II diseased furcations

The tunnel technique. This is mainly used to treat advanced Grade II and Grade III lesions $(3,9)$. It requires that the root trunks be short, and that there be wide root divergence and a favorable crown/root ratio. Nowadays the technique has lost much of its validity given the difficulties it causes with respect to maintaining adequate hygiene, especially of the upper molars. The aim of the technique is to permit the access of an interproximal brush to the fornix of the furcation; basically the furcation lesion is made larger to allow better hygiene, but few patients manage to maintain an adequate cleaning routine.

The main risks associated with this treatment include the covering of the tunnel by gingival growth (induced by inflammation caused by plaque), the appearance of secondary caries, the generation of thermal hyperesthesia, the need to undertake endodontic treatment due to the presence of an accessory canal, and the need for osteotomy. Tunneling commonly creates an ecological habitat that is difficult to keep clean: food remains and bacteria therefore accumulate, generating inflammation.

Guided tissue regeneration. Guided tissue regeneration (GTR) has been shown to be useful in the treatment of Grade II lesions (10). However, it should be remembered that spontaneous regeneration following meticulous debridement is also possible. The commercial availability of bone morphogenetic proteins (BMP) and amelogenetic protein matrix has provided a new focus for this kind of treatment. These products can be used alone or in combination.

Unfortunately, the cost/benefit ratio for these techniques is poor - the price a patient has to pay for every millimeter regenerated being very high. The possibility of failure due to the exposure of the surgical membrane or infection (11) must also be taken into account. Some authors suggest that the use of bone substitution material improves the outcome of this type of treatment, while others report no improvement over the use of surgical membranes alone.

- Grade III diseased furcations

Guided tissue regeneration. This is of very little use in the treatment of such advanced grade disease. It should only be attempted with lesions that are under control, isolated, and when the patients can easily maintain hygiene. The outcome, however, is very uncertain $(10,12)$. Root hemisection. This consists of individualization of the roots of a multi-root molar. The technique is generally performed on the lower molars; only exceptionally is it attempted on the upper molars. Prior endodontic treatment is always necessary. Later restoration can be 
attempted using a single crown prosthesis (a de facto tunnel prosthesis). However, it is more effective and practical to perform premolarization of the roots followed by their individualization.

The main difficulties associated with this treatment include a lack of inter-root space, convergence or fusion of the root, cracking of the roots due to overloading, and a lack of bone support (an unfavorable crown/root ratio)(2).

Root amputation. This consists of eliminating one of the roots, generally that which provides the least support or that which, due to its proximity, may compromise the stability of a neighboring tooth. As in hemisection, prior endodontic treatment is necessary, and a minimum of 2-3 months should be allowed to elapse in order to confirm the success of the treatment (2). For the amputation itself, a flap is raised, with separation being performed in the mesial to distal and apical to coronal directions. Amputation may involve part of the crown.

In the upper molars, the distovestibular root has poor bone support. In addition, it is inclined and often interferes with the mesial root of the second upper molar. It is therefore usually the root of choice for elimination. However, this root is strong, and inserts in line with the premolars, allowing it to receive the occlusal load in an axial direction. The palatine root usually has a single canal which is easily accessed, but its palatine angle means it receives the occlusal load poorly. On occasion, however, it is the only option (13). The removal of one root of a maxillary molar does not increase the mobility of the tooth under conditions of normal function, and the routine splinting of root-resected teeth does not seem to be indicated (14).

In the lower molars, the mesial root can fracture more easily than the distal root. However, a single root is insufficient to maintain the occlusion of a lower molar, and support must be sought from the anterior premolar if the distal root is preserved, or from the posterior molar if the mesial root is preserved. Another alternative is to use an implant to provide the support lost from elimination of the root.

The upper premolars, canines and other teeth with furcations are not usually candidates for this kind of treatment, since they would be left with too little support.

Extraction. Heroic efforts to maintain a tooth with a furcation affected by serious periodontal disease need to be reconsidered. In some cases this might provide no clear benefit and may even be worse for the patient. The loss of bone that may accompany desperate efforts to preserve a tooth may limit later therapeutic options such as substitution with an implant. Extraction should be considered when the treatment of a particularly loose tooth is likely to be complex and costly.

\section{Prognosis}

A number of studies confirm that about $85 \%$ of all teeth treated for periodontal disease affecting the furcation can be maintained stable for 3-7 years. Thus, if the patient can be trusted to maintain adequate hygiene, and treatment appropriate for the tooth in question has been effective, an affected tooth may be preserved for a prolonged period of time (15). This may be acceptable to both the patient and dental professional.

\section{References}

1. Bascones-Martínez A, Figuero-Ruiz E. Periodontal diseases as bacterial infection. Med Oral Patol Oral Cir Bucal. 2004;9 Suppl:101-7, 92-100.

2. DeSanctis M, Murphy KG. The role of resective periodontal surgery in the treatment of furcation defects. Periodontol 2000. 2000;22:154-68.

3. Cattabriga M, Pedrazzoli V, Wilson TG Jr. The conservative approach in the treatment of furcation lesions. Periodontol 2000. 2000;22:133-53

4. Papapanou PN, Tonetti MS. Diagnosis and epidemiology of periodontal osseous lesions. Periodontol 2000. 2000;22:8-21.

5. Svärdström G, Wennström JL. Prevalence of furcation involvements in patients referred for periodontal treatment. J Clin Periodontol. 1996;23:1093-9.

6. Hamp SE, Nyman S, Lindhe J. Periodontal treatment of multirooted teeth. Results after 5 years. J Clin Periodontol. 1975;2:126-35.

7. Armitage GC, Research, Science and Therapy Committee of the American Academy of Periodontology. Diagnosis of periodontal diseases. J Periodontol. 2003;74:1237-47.

8. McClain PK, Schallhorn RG. Focus on furcation defects--guided tissue regeneration in combination with bone grafting. Periodontol 2000. 2000;22:190-212.

9. Rüdiger SG. Mandibular and maxillary furcation tunnel preparations--literature review and a case report. J Clin Periodontol. 2001;28:1-8.

10. Karring T, Cortellini P. Regenerative therapy: furcation defects. Periodontol 2000. 1999;19:115-37.

11. De Sanctis M, Zucchelli G, Clauser C. Bacterial colonization of barrier material and periodontal regeneration. J Clin Periodontol. 1996;23:1039-46.

12. Sanz M, Giovannoli JL. Focus on furcation defects: guided tissue regeneration. Periodontol 2000. 2000;22:169-89.

13. Ross IF, Thompson RH Jr. Furcation involvement in maxillary and mandibular molars. J Periodontol. 1980;51:450-4.

14. Klavan B. Clinical observations following root amputation in maxillary molar teeth. J Periodontol. 1975;46:1-5.

15. Basten CH, Ammons WF Jr, Persson R. Long-term evaluation of root-resected molars: a retrospective study. Int J Periodontics Restorative Dent. 1996;16:206-19. 